\title{
A Method for Assimilation of Lagrangian Data
}

\author{
L. KUZNETSOV \\ Department of Mathematics, University of North Carolina at Chapel Hill, Chapel Hill, North Carolina
}

K. IDE

Department of Atmospheric Sciences and Institute of Geophysics and Planetary Physics, University of California, Los Angeles, Los Angeles, California

C. K. R. T. JONES

Department of Mathematics, University of North Carolina at Chapel Hill, Chapel Hill, North Carolina

(Manuscript received 8 November 2002, in final form 4 April 2003)

\section{ABSTRACT}

\begin{abstract}
Difficulties in the assimilation of Lagrangian data arise because the state of the prognostic model is generally described in terms of Eulerian variables computed on a fixed grid in space, as a result there is no direct connection between the model variables and Lagrangian observations that carry time-integrated information. A method is presented for assimilating Lagrangian tracer positions, observed at discrete times, directly into the model. The idea is to augment the model with tracer advection equations and to track the correlations between the flow and the tracers via the extended Kalman filter. The augmented model state vector includes tracer coordinates and is updated through the correlations to the observed tracers.

The technique is tested for point vortex flows: an $N_{F}$ point vortex system with a Gaussian noise term is modeled by its deterministic counterpart. Positions of $N_{D}$ tracer particles are observed at regular time intervals and assimilated into the model. Numerical experiments demonstrate successful system tracking for $\left(N_{F}, N_{D}\right)=$ $(2,1),(4,2)$, provided the observations are reasonably frequent and accurate and the system noise level is not too high. The performance of the filter strongly depends on initial tracer positions (drifter launch locations). Analysis of this dependence shows that the good launch locations are separated from the bad ones by Lagrangian flow structures (separatrices or invariant manifolds of the velocity field). The method is compared to an alternative indirect approach, where the flow velocity, estimated from two (or more) consecutive drifter observations, is assimilated directly into the model.
\end{abstract}

\section{Introduction}

Lagrangian meters, such as weather balloons and ocean drifters and floats, provide a substantial part of atmospheric and oceanic data. These data are used to reconstruct mean large-scale currents, estimate the rate of relative dispersion, and give insight into the formation, movement, and interaction of coherent flow features, such as eddies, etc. (Dutkiewicz et al. 2001; Garfield et al. 2001; Carr and Rossby 2001; Bower and Hunt 2000). Trajectories of Lagrangian tracers also contain detailed quantitative information about the dynamics of the underlying flow, but this information is currently not used for assimilation into flow models. The reason is that most assimilation schemes in oceanography and meteorology use model variables computed

Corresponding author address: Dr. Leonid Kuznetsov, Department of Mathematics, University of North Carolina at Chapel Hill, CB\#3250, Phillips Hall, Chapel Hill, NC 27599-3250.

E-mail: leonid@email.unc.edu on a fixed grid in space (Ghil and Malanotte-Rizzoli 1991), whereas the Lagrangian observations are distributed nonuniformly over the space and do not give the data directly in terms of model variables.

In this work we present a new method for the assimilation of Lagrangian (drifter) data that follows a sequential approach. Our approach is based on applying the extended Kalman filter (EKF; Jazwinski 1970) to the dynamics in the augmented state space that includes drifter coordinates as extra variables (Ide et al. 2002). The augmented state vector $\mathbf{x}=\left(\mathbf{x}_{F}, \mathbf{x}_{D}\right)$ combines the Eulerian part $\mathbf{x}_{F}$ describing the state of the flow, and the Lagrangian part $\mathbf{x}_{D}$-coordinates of the drifters. Advection equations for $\mathbf{x}_{D}$ are added to the model (equations for the evolution of the Eulerian variables are not changed). The augmented error covariance matrix is evolved by the tangent linear model (TLM). On the level of second-order statistics it carries all necessary information including the correlations between the errors in flow variables and the errors in drifter positions. Due 
to the correlations, the assimilation of the tracer information corrects not only the tracer part of the state vector $\mathbf{x}_{D}$ but also the flow variables $\mathbf{x}_{F}$. The correction vector Kd is a product of the innovation vector $\mathbf{d}$ (differences between observed drifter coordinates and their predictions by the model) and the Kalman gain matrix K. The latter has dimension $N+L$ by $L$, where $N, L$ are dimensions of $\mathbf{x}_{F}, \mathbf{x}_{D}$. The first $N$ rows of the matrix $\mathbf{K}$ give the weights with which drifter observations correct the state variables, they are proportional to the part of the error covariance matrix corresponding to correlations between the flow state and drifter positions. These correlations always appear because drifter motion depends on the underlying flow. A different approach where drifter data were used to derive Eulerian (velocity) information that is assimilated into the model was used in Özgökmen et al. (2000, 2003, manuscript submitted to J. Geophys. Res.); Molcard et al. (2003).

The advantage of the proposed scheme is that including drifters into the dynamical model and tracking them and their correlations with the flow numerically allows us to extract maximal information about the flow from drifter observations. This is achieved by computing dynamically consistent weights (given by the gain matrix K) at each update step, so that the new information from the observations is distributed according to the actual structure of the flow.

To test the technique we apply it to point vortex flows. The model is given by a system of $N_{F}$ point vortices in a plane $\left(N=2 N_{F}\right)$, and the actual flow (model truth) is governed by the same system but with a stochastic white noise term added that represents unresolved smallscale processes. Positions of $N_{D}$ passively advected particles (tracers) are observed at regular time intervals and assimilated into the model; further, no observations of vortices are made $\left(L=2 N_{D}\right)$. The flows with $N_{F}=2$ (model vortices exhibit regular motion) and $N_{F}=4$ [model vortices in chaotic or (quasi) periodic regimes] were considered. We have found that, provided the observations are frequent and accurate enough, the assimilating model stays close to the original (stochastic) one, that is, by observing and assimilating only a few tracer positions $\left(N_{D}=1,2\right)$ it was possible to track both tracers and vortices.

When the observations are taken less frequently or when the noise levels are increased the scheme is prone to abrupt divergence caused by the passage of tracers through the neighborhoods of saddle-points for the velocity field. The cause of this filter malfunction is the breakdown of the TLM (that is used to predict the model error covariance matrix) due to the fast exponential error growth in the vicinity of the saddle-point. This problem is connected with the difficulties, caused by an exponential separation rate in the vicinity of unstable critical points, which, in its Eulerian analog, is known as a cause of divergence of the EKF (Ide and Ghil 1998; Miller et al. 1994; Evensen 1997; Miller et al. 1999; Verlaan and Heemink 2001).
The successful performance of the above method in the case of this simple (although strongly nonlinear) system gives a favorable perspective on the possibility of applying it to realistic oceanographic models. One of the challenges in applying the EKF to these models is the forecasting of the error covariance matrix, which has very large dimension (square of the number of degrees of freedom of the entire model). Forecasting it by direct solution of the evolution equation becomes computationally too expensive. Modifications of the EKF such as various versions of the ensemble Kalman filter (Anderson 2001; Evensen 1994; Heemink et al. 2001; Pham 2001) may be employed to overcome this problem. Another fundamental problem is the sparsity of oceanographic data. Although part of the attraction of Lagrangian observations lies in the relatively low cost of drifters and floats compared to moorings (and ships) that collect Eulerian data, it is still too expensive to cover the ocean densely. Therefore, finding where and when a drifter should be placed to reveal as much information as possible about the flow that drives it is a question of considerable practical importance (Poje et al. 2002).

The rest of the paper is organized as follows. In section 2, we review extended Kalman filtering in order to establish notation and introduce the assimilation scheme for the augmented model that includes the flow and the drifters. The point vortex model is described in section 3. Section 4 contains our computational results: examples of successful tracking in two- and four-vortex systems, analysis of the filter divergence, dependence of the efficiency of the scheme on the drifter launch location, and comparison of our scheme with an alternative indirect method based on the assimilation of the flow velocity derived from consecutive drifter observations.

\section{Extended Kalman filter for Lagrangian data}

In numerical models the state of the flow is represented as an $N$-dimensional vector $\mathbf{x}(t)$, which is obtained by a discretization of the model equations and comprises all relevant dynamical variables, for example, flow velocity, density, etc. The dimension of the state vector $N$ is a product of the number of these variables and the number of discretization elements (grid points, Fourier modes, etc.), and therefore is typically large. The model describing the evolution of the state vector is a deterministic equation

$$
\frac{d \mathbf{x}^{f}}{d t}=M\left(\mathbf{x}^{f}, t\right),
$$

where $M$ is the corresponding dynamics operator. The superscript $f$ (forecast) distinguishes the solution of (1) from the value of the state vector variables in the true flow $\mathbf{x}^{t}$ (Ide et al. 1997). A closed system of equations for $\mathbf{x}^{t}$ is usually unavailable because the state variables 
interact with the subgrid-scale processes that are not represented by the state vector.

The system (1) might be our best guess for a deterministic equation for $\mathbf{x}^{t}$, but we can go further if something is known about the statistics of the small-scale processes. Then the discretized dynamics of $\mathbf{x}^{t}$ can be written as a stochastic system

$$
d \mathbf{x}^{t}=M\left(\mathbf{x}^{t}, t\right) d t+\boldsymbol{\eta}^{t}(t) d t .
$$

The noise term $\boldsymbol{\eta}^{t}(t)$ represents the effect of the unresolved small scales, and we assume below that it is a zero-mean Gaussian white noise:

$E\left[\boldsymbol{\eta}^{t}(t)\right]=0, \quad E\left\{\boldsymbol{\eta}^{t}(t)\left[\boldsymbol{\eta}^{t}\left(t^{\prime}\right)\right]^{\mathrm{T}}\right\}=\delta\left(t-t^{\prime}\right) \mathbf{Q}^{t}(t)$

Sequential data assimilation updates the model every time an observation of the true state becomes available. The observations at $t_{j}$ can be written in terms of $\mathbf{x}_{j}^{t} \equiv$ $\mathbf{x}^{t}\left(t_{j}\right)$ as

$$
\mathbf{y}_{j}^{o}=\mathbf{H}_{j}\left[\mathbf{x}_{j}^{t}\right]+\boldsymbol{\epsilon}_{j}^{t},
$$

where $\mathbf{H}_{j}$ is a vector of observation functions (forward observation operators) and $\boldsymbol{\epsilon}_{j}^{t}$ are random variables representing errors of the observations, which are typically assumed to be uncorrelated zero-mean Gaussian with a covariance

$$
E\left[\boldsymbol{\epsilon}_{j}^{t}\left(\boldsymbol{\epsilon}_{m}^{t}\right)^{\mathrm{T}}\right]=\delta_{j m} \mathbf{R}_{j}^{t}
$$

The dimension of $\boldsymbol{y}_{j}^{o}$ is equal to the number of observations $L_{j}$ available at $t_{j}$. The number and type of observations can vary at each update time.

To combine the observations with the model prediction we will use the extended Kalman filter. A key element of the EKF is tracking the evolution of the model error covariance matrix

$$
\mathbf{P}^{f} \equiv E\left[\left(\mathbf{x}^{f}-\mathbf{x}^{t}\right)\left(\mathbf{x}^{f}-\mathbf{x}^{t}\right)^{\mathrm{T}}\right]
$$

using the TLM, which gives a closed equation for $\mathbf{P}^{f}$ :

$$
\left.\frac{d \mathbf{P}^{f}}{d t}=\mathbf{M} \mathbf{P}^{f}+(\mathbf{M P})^{f}\right)^{\mathrm{T}}+\mathbf{Q}(t)
$$

where

$$
\mathbf{M} \equiv \frac{\partial M\left(\mathbf{x}^{t}, t\right)}{\partial \mathbf{x}}
$$

is the linearized dynamics operator, evaluated at $\mathbf{x}^{f}(t)$, and $\mathbf{Q}(t)$ is our estimation for system noise covariance $\mathbf{Q}^{t}(t)$.

At each update time $t_{j}$, a new state vector $\mathbf{x}_{j}^{a}$, called the analysis state, is sought such that it minimizes the mean-square error

$$
\operatorname{tr}_{j}^{a}=E\left[\left(\mathbf{x}_{j}^{a}-\mathbf{x}_{j}^{t}\right)^{\mathrm{T}}\left(\mathbf{x}_{j}^{a}-\mathbf{x}_{j}^{t}\right)\right] .
$$

The EKF gives a first-order approximation to an optimum analysis state using the model error covariance matrix predicted by the linearized Eq. (7). The update

$$
\mathbf{x}_{j}^{a}=\mathbf{x}_{j}^{f}+\mathbf{K}_{j} \mathbf{d}_{j}
$$

is a linear combination of the predicted model state vector $\mathbf{x}_{j}^{f}$ and the product of the innovation vector (the $L_{j}$ dimensional vector of differences between the observations and the prediction of the observed quantities by the model)

$$
\mathbf{d}_{j} \equiv \mathbf{y}_{j}^{o}-\mathbf{H}_{j}\left[\mathbf{x}_{j}^{f}\right]
$$

and the Kalman gain matrix $\mathbf{K}_{j}$ (dimension $N \times L_{j}$ )

$$
\mathbf{K}_{j}=\mathbf{P}_{j}^{f} \mathbf{H}_{j}^{\mathrm{T}}\left(\mathbf{H}_{j} \mathbf{P}_{j}^{f} \mathbf{H}_{j}^{\mathrm{T}}+\mathbf{R}_{j}\right)^{-1} .
$$

Here

$$
\mathbf{H}_{j}=\frac{\partial \mathbf{H}_{j}}{\partial \mathbf{x}}
$$

is the linearized observation matrix and $\mathbf{R}_{j}$ is our estimation of the the covariance matrix of the observation error (5). The updated error covariance matrix is given by

$$
\mathbf{P}_{j}^{a}=\left(\mathbf{I}-\mathbf{K}_{j} \mathbf{H}_{j}\right) \mathbf{P}_{j}^{f} .
$$

In this study, we consider the case when the observations are provided by Lagrangian tracers. Positions of $N_{D}$ tracers $\mathbf{x}_{D}$ are observed at $t_{j}\left(L_{j}=2 N_{D} \equiv L\right)$. Note that an observation of tracer positions at one time does not give any information about the flow, it will contain such information only as the tracer position is also known at some previous time $t_{k}, k<j$. The new positions $\mathbf{x}_{D}\left(t_{j}\right)$ depend on $\mathbf{x}_{D}\left(t_{k}\right)$ and on the flow on $\left[t_{k}\right.$, $\left.t_{j}\right]$.

To extract the information about the flow from the tracer observations we augment the model state space, so that the new model state vector

$$
\mathbf{x}=\left(\begin{array}{l}
\mathbf{x}_{F} \\
\mathbf{x}_{D}
\end{array}\right)
$$

combines the state vector of the flow $\mathbf{x}_{F}$ and the vector of the tracer coordinates $\mathbf{x}_{D}$. Tracer advection equations are added to the model:

$$
\begin{aligned}
\frac{d \mathbf{x}_{F}^{f}}{d t} & =M_{F}\left(\mathbf{x}_{F}^{f}, t\right), \\
\frac{d \mathbf{x}_{D}^{f}}{d t} & =M_{D}\left(\mathbf{x}_{D}^{f}, \mathbf{x}_{F}^{f}, t\right) .
\end{aligned}
$$

The first part of (16) is the unchanged original model (1), the second part represents discretized tracer advection equations. The linearized dynamics operator (8) and the error covariance matrix are $(N+L) \times(N+L)$ matrices that can be written in a block form as

$$
\begin{aligned}
\mathbf{M} & =\left(\begin{array}{cc}
\mathbf{M}_{F F} & 0 \\
\mathbf{M}_{D F} & \mathbf{M}_{D D}
\end{array}\right), \quad \text { and } \\
\mathbf{P} & =\left(\begin{array}{ll}
\mathbf{P}_{F F} & \mathbf{P}_{F D} \\
\mathbf{P}_{D F} & \mathbf{P}_{D D}
\end{array}\right),
\end{aligned}
$$

where $\mathbf{M}_{F F}$ and $\mathbf{P}_{F F}$ are $N$ by $N$ matrices, $\mathbf{M}_{D D}$ and $\mathbf{P}_{D D}$ are $L \times L$, etc. In practice the number of available observations at each update time is much less than the 
number of degrees of freedom of the model, $L \ll N$, which presents a challenge for successful tracking of the flow. On the other hand, it means that the increase in the dimension of the model, and therefore in the computational cost of dealing with a bigger system, is relatively small.

The observation function $\mathbf{H}$ corresponding to tracer positions is linear:

$$
\mathbf{H}_{j}\left[\mathbf{x}_{j}^{t}\right]=\mathbf{H} \mathbf{x}_{j}^{t}, \quad \mathbf{H}=(\mathbf{0} \mathbf{I}),
$$

where $\mathbf{0}$ is a $L \times N$ matrix of zeros, and I is a $L \times L$ unit matrix. This results in a special form of the Kalman gain matrix:

$$
\mathbf{K}=\left(\begin{array}{l}
\mathbf{P}_{F D} \\
\mathbf{P}_{D D}
\end{array}\right)\left(\mathbf{P}_{D D}+\mathbf{R}\right)^{-1} .
$$

The weights with which drifter observations correct the flow variables $\mathbf{x}_{F}$ are given by the first $N$ rows of $\mathbf{K}$, they are proportional to $\mathbf{P}_{F D}$, the correlations between the flow state and the drifter positions. These correlations always appear because drifter paths depend on the flow, $\mathbf{M}_{D F} \neq 0$. Note that $\mathbf{P}_{F F}$, which is the largest part of the error covariance matrix, does not enter into (20). It is required, however, for the prediction of $\mathbf{P}_{F D}$, since (7) couples all blocks of $\mathbf{P}$. Nevertheless, the absence of the direct dependence of the Kalman gain on $\mathbf{P}_{F F}$ suggests that some simplified form of the latter could be used (Carter 1989; Cohn 1993).

\section{Point vortex systems}

Point vortex flows are singular solutions to 2D Euler's equation. They are often used to model 2D flows dominated by strong coherent vortices (McWilliams 1990; Babiano et al. 1994; Weiss et al. 1998). A flow due to $N_{F}$ point vortices is specified by their positions on the plane $\left[x_{m}(t), y_{m}(t)\right], m=1, \ldots, N_{F}$, so the state vector has dimension $N=2 N_{F}$. We will use complex coordinates $z_{m}(t)=x_{m}(t)+i y_{m}(t)$, and a complex-valued state vector $\mathbf{z} \in \mathbf{C}^{N_{F}}$. The dynamics of vortices is then governed by the equations (Friedrichs 1966; Aref and Pomphrey 1982):

$$
\begin{gathered}
\dot{z}_{m}^{t}=\frac{i}{2 \pi} \sum_{l=1, l \neq m}^{N_{F}} \frac{\Gamma_{l}}{z_{m}^{t *}-z_{l}^{t *}}+\eta_{F m}(t), \\
\left(m=1, \ldots, N_{F}\right),
\end{gathered}
$$

where $\Gamma_{l}$ is circulation of vortex $l$, and the stochastic term $\boldsymbol{\eta}_{F} \in \mathbf{C}^{N_{F}}, \boldsymbol{\eta}_{F}=\boldsymbol{\eta}_{F}^{(x)}+i \boldsymbol{\eta}_{F}^{(y)}$ is added to represent unresolved processes. In Ide and Ghil (1998) such a system was modeled by its deterministic part, and observations of the vortex positions and of flow velocities at fixed stations were assimilated using the EKF.

Below we will consider the case when the data is provided by observations of passive tracers, and assimilate this information using the method introduced in the previous section. The tracers are advected according to

$$
\begin{gathered}
\dot{\zeta}_{m}^{t}=\frac{i}{2 \pi} \sum_{l=1}^{N_{F}} \frac{\Gamma_{l}}{\zeta_{m}^{* *}-z_{l}^{t *}}+\eta_{D m}(t), \\
\left(m=1, \ldots, N_{D}\right),
\end{gathered}
$$

where $N_{D}$ is the total number of tracers, $\zeta \in \mathbf{C}^{N_{D}}$ are their complex coordinates, $\boldsymbol{\eta}_{D}=\boldsymbol{\eta}_{D}^{(x)}+i \boldsymbol{\eta}_{D}^{(y)}$ are system noise.

In this study, we take $\boldsymbol{\eta}_{F}$ and $\boldsymbol{\eta}_{D}$ to be given by uncorrelated, white Gaussian noise with zero mean $E\left[\boldsymbol{\eta}_{F}^{(x, y)}\right]=E\left[\boldsymbol{\eta}_{D}^{(x, y)}\right]=0$, and covariance given, respectively, by

$$
\begin{array}{lr}
E\left[\boldsymbol{\eta}_{F}^{(x, y)}(t) \boldsymbol{\eta}_{F}^{(x, y)}\left(t^{\prime}\right)\right]=\sigma^{2} \delta\left(t-t^{\prime}\right) \mathbf{I}, & E\left[\boldsymbol{\eta}_{F}^{(x)} \boldsymbol{\eta}_{F}^{(y)}\right]=0 \\
E\left[\boldsymbol{\eta}_{D}^{(x, y)}(t) \boldsymbol{\eta}_{D}^{(x, y)}\left(t^{\prime}\right)\right]=\sigma^{2} \delta\left(t-t^{\prime}\right) \mathbf{I}, & E\left[\boldsymbol{\eta}_{D}^{(x)} \boldsymbol{\eta}_{D}^{(y)}\right]=0 .
\end{array}
$$

System noise is assumed to lead to the same covariance for vortices and tracers.

We form the state vector describing both the flow and the tracers:

$$
\mathbf{x}=\left(\begin{array}{l}
\mathbf{z} \\
\boldsymbol{\zeta}
\end{array}\right), \quad \mathbf{x} \in \mathbf{C}^{N_{F}+N_{D}} .
$$

The forecast state $\mathbf{x}^{f}$ is computed by solving the deterministic part of (21) and (22). The error $\Delta \mathbf{x}^{f} \equiv \mathbf{x}^{f}-\mathbf{x}^{t}$ is assumed to have a zero mean, its covariance is represented as a $2\left(N_{F}+N_{D}\right) \times 2\left(N_{F}+N_{D}\right)$ matrix

$$
\begin{aligned}
\mathbf{P} & \equiv\left(\begin{array}{ll}
\mathbf{P}_{1} & \mathbf{P}_{2} \\
\mathbf{P}_{2}^{*} & \mathbf{P}_{1}^{*}
\end{array}\right), \quad \mathbf{P}_{1}=E\left[\Delta \mathbf{x} \Delta \mathbf{x}^{\mathrm{T}}\right], \\
\mathbf{P}_{2} & =E\left[\Delta \mathbf{x} \Delta \mathbf{x}^{* \mathrm{~T}}\right]
\end{aligned}
$$

(unbiased assumption is generally violated in nonlinear models; it is our assumption that $\Delta \mathbf{x}^{f}$ remains small, and the bias can be neglected). The TLM gives

$$
\dot{\mathbf{P}}=\mathbf{M}_{2} \mathbf{P}+\mathbf{P}^{\mathrm{T}} \mathbf{M}_{2}^{\mathrm{T}}+\mathbf{Q},
$$

where the linearized dynamics operator $\mathbf{M}_{2}$ is obtained by differentiating the model equations (21) and (22) and their complex conjugates with respect to $\mathbf{x}$ and $\mathbf{x}^{*}$ :

$$
\begin{aligned}
\mathbf{M}_{2}= & \left(\begin{array}{cc}
0 & \mathbf{M} \\
\mathbf{M}^{*} & 0
\end{array}\right), \quad \mathbf{M}=\left(\begin{array}{cc}
\mathbf{M}_{F F} & 0 \\
\mathbf{M}_{D F} & \mathbf{M}_{D D}
\end{array}\right), \\
\mathbf{M}_{F F, m l} \equiv & \left(1-\delta_{m l}\right) \frac{i \Gamma_{l}}{2 \pi\left(z_{m}^{f *}-z_{l}^{f *)^{2}}\right.} \\
& -\delta_{m l} \sum_{k \neq m, k=1}^{N_{F}} \frac{i \Gamma_{k}}{2 \pi\left(z_{m}^{f *}-z_{k}^{f *}\right)^{2}} \\
\mathbf{M}_{D F, m l} \equiv & \frac{i \Gamma_{l}}{2 \pi\left(\zeta_{m}^{f *}-z_{l}^{f *}\right)^{2}}, \\
\mathbf{M}_{D D, m l} \equiv & \delta_{m l} \sum_{k=1}^{N} \frac{i \Gamma_{k}}{2 \pi\left(\zeta_{m}^{f *}-z_{k}^{f *}\right)^{2}} .
\end{aligned}
$$


The system noise covariance matrix $\mathbf{Q}$ is [from (23) and (24)]:

$$
\mathbf{Q}=2 \sigma^{2}\left(\begin{array}{ll}
0 & \mathbf{I} \\
\mathbf{I} & 0
\end{array}\right)
$$

Due to the symmetry of the error covariance matrix $\left(\mathbf{P}_{1}\right.$ $\left.=\mathbf{P}_{1}^{\mathrm{T}}, \mathbf{P}_{2}^{*}=\mathbf{P}_{2}^{\mathrm{T}}\right)$, the system (27) splits into two coupled $\left(N_{F}+N_{D}\right) \times\left(N_{F}+N_{D}\right)$ systems

$$
\begin{aligned}
& \dot{\mathbf{P}}_{1}=\mathbf{M} \mathbf{P}_{2}^{\mathrm{T}}+\mathbf{P}_{2} \mathbf{M}^{\mathrm{T}}, \\
& \dot{\mathbf{P}}_{2}=\mathbf{M} \mathbf{P}_{1}^{*}+\mathbf{P}_{1} \mathbf{M}^{* \mathrm{~T}}+2 \sigma^{2} \mathbf{I}
\end{aligned}
$$

The tracer positions are measured at regular time intervals $t_{j}=j \Delta T, j=1,2, \ldots$, providing $L=2 N_{D}$ independent observations:

$$
\mathbf{y}_{j}^{O}=\mathbf{H}\left[\mathbf{x}^{t}\left(t_{j}\right)\right]+\boldsymbol{\epsilon}_{j}=\boldsymbol{\zeta}_{j}^{t}+\boldsymbol{\epsilon}_{j}, \quad \mathbf{y}_{j}^{O} \in \mathbf{C}^{N_{D}},
$$

that is, the observation function (19) is

$$
\mathbf{H}\left(\mathbf{x}^{t}\right)=\mathbf{H} \mathbf{x}^{t}, \quad \mathbf{H}=(\mathbf{0} \mathbf{I}),
$$

where $\mathbf{0}$ is $N_{D} \times N_{F}$ and $\mathbf{I}$ is $N_{D} \times N_{D}$.

In our simulations the observation errors $\boldsymbol{\epsilon}_{j}=\boldsymbol{\epsilon}_{j}^{(x)}+$ $i \boldsymbol{\epsilon}_{j}^{(y)}$ are distributed as independent Gaussians with the same statistics:

$$
\begin{aligned}
E\left[\boldsymbol{\epsilon}_{j}^{(x)} \boldsymbol{\epsilon}_{j}^{(x)}\right] & =E\left[\boldsymbol{\epsilon}_{j}^{(y)} \boldsymbol{\epsilon}_{j}^{(y)}\right]=\rho^{2} \mathbf{I}, \\
E\left[\boldsymbol{\epsilon}_{j}^{(x)}\right] & =E\left[\boldsymbol{\epsilon}_{j}^{(y)}\right]=E\left[\boldsymbol{\epsilon}_{j}^{(x)} \boldsymbol{\epsilon}_{j}^{(y)}\right]=0 .
\end{aligned}
$$

At each observation, the state vector is updated according to (10), where the $\mathbf{x}$ and $\mathbf{y}$ are extended to include their complex conjugates. Using the block form (26) the update can be written as

$$
\mathbf{x}^{a}=\mathbf{x}^{f}+\mathbf{K}_{1}\left(\mathbf{y}^{O}-\mathbf{H} \mathbf{x}^{f}\right)+\mathbf{K}_{2}\left(\mathbf{y}^{O}-\mathbf{H} \mathbf{x}^{f}\right)^{*},
$$

where the subscript $j$ is dropped for simplicity in notation.

The error covariance matrix is updated according to (14) or in terms of $\mathbf{P}_{1}$ and $\mathbf{P}_{2}$

$$
\begin{aligned}
& \mathbf{P}_{1}^{a}=\mathbf{I}-\mathbf{K}_{1} \mathbf{H P}_{1}^{f}-\mathbf{K}_{2} \mathbf{H} \mathbf{P}_{2}^{f} *, \\
& \mathbf{P}_{2}^{a}=\mathbf{K}_{1} \mathbf{H P}_{2}^{f}-\mathbf{K}_{2} \mathbf{H P}_{1}^{f} * .
\end{aligned}
$$

The Kalman gain matrix is given by

$$
\mathbf{K}=\left(\begin{array}{ll}
\mathbf{K}_{1} & \mathbf{K}_{2} \\
\mathbf{K}_{2}^{*} & \mathbf{K}_{1}^{*}
\end{array}\right)=\mathbf{P}_{f} \mathbf{H}_{2}^{\mathrm{T}}\left(\mathbf{H}_{2} \mathbf{P}^{f} \mathbf{H}_{2}^{\mathrm{T}}+\mathbf{R}\right)^{-1},
$$

where $\mathbf{H}_{2}$ is the $2 L \times 2(N+L)$ extended observation matrix

$$
\begin{aligned}
\mathbf{H}_{2} & =\left(\begin{array}{cc}
\mathbf{H} & 0 \\
0 & \mathbf{H}
\end{array}\right) \text { and } \\
\mathbf{R} & =\left[\begin{array}{cc}
E\left(\boldsymbol{\epsilon} \boldsymbol{\epsilon}^{\mathrm{T}}\right) & E\left(\boldsymbol{\epsilon}^{* \mathrm{~T}}\right) \\
E\left(\boldsymbol{\epsilon}^{* \mathrm{~T}} \boldsymbol{\epsilon}\right) & E\left(\boldsymbol{\epsilon}^{*} \boldsymbol{\epsilon}^{* \mathrm{~T}}\right)
\end{array}\right]=2 \rho^{2}\left(\begin{array}{ll}
0 & \mathbf{I} \\
\mathbf{I} & 0
\end{array}\right)
\end{aligned}
$$

is the covariance matrix of the observation error. The blocks of the Kalman matrix, $\mathbf{K}_{1}$ and $\mathbf{K}_{2}$, have the same structure as in (20): their first $N_{F}$ rows, that is, the weights with which the vortex coordinates are updated, are proportional to the correlations between the vortices and the tracers (off-diagonal blocks of $\mathbf{P}_{1}$ and $\mathbf{P}_{2}$ ).

\section{Computational examples}

\section{a. Two vortices}

We start from the case of two identical vortices with circulations $\Gamma_{1}=\Gamma_{2} \equiv \Gamma$, and initial positions $z_{1}(0)=$ $-1, z_{2}(0)=1$. We take $\Gamma=2 \pi$, for different $\Gamma, l \equiv$ $\left|z_{1}-z_{2}\right|$ the scaling of coordinates and time

$$
x \rightarrow 2 x / l, \quad t \rightarrow\left(2 \Gamma / \pi l^{2}\right) t,
$$

would recover our parameters; the system noise and observation error variances would scale as $\sigma \rightarrow\left(\Gamma^{1 / 2} /\right.$ $2 \pi) \sigma$ and $\rho \rightarrow 2 \rho / l$.

When the stochastic forcing is present or the initial conditions are imprecise, the deterministic model loses track of the full system, that is, after some time $\tau$ the error in vortex positions grows up to the order of the system size $l$. The breakdown time $\tau$ can be estimated from the covariance Eq. (7). For two identical vortices with

$$
\begin{aligned}
E\left[z_{1,2}^{t}(0)\right] & =z_{1,2}(0)= \pm 1 \quad \text { and } \\
E\left[\Delta z_{m}(0) \Delta z_{l}^{*}(0)\right] & =\delta_{m l} 2 \rho^{2}, \quad m, l=1,2
\end{aligned}
$$

the model solution is $z_{1,2}^{f}(t)= \pm \exp i \omega t,(\omega=1 / 2$, period $T=4 \pi$ ), and the solution of the variance Eq. (27) gives

$$
\begin{aligned}
E & {\left[\Delta z_{m}(t) \Delta z_{m}^{*}(t)\right] } \\
& =2\left(\rho^{2}+\sigma^{2} t+\rho^{2} \omega^{2} t^{2}+\frac{\sigma^{2} \omega^{2} t^{3}}{3}\right) .
\end{aligned}
$$

Our simulations have $\sigma$ and $\rho$ in the range $0.01-0.5$, and the model loses track of the full system in one-two motion periods. The covariance Eq. (27) becomes a poor approximation for the error propagation for $t<\tau$ (nonlinear terms become important when $\left|z^{f}-z^{t}\right| \sim l$ ) and therefore (44) gives only a rough estimation for the breakdown time.

To track vortex positions for an extended period of time new information about the full system has to be injected into the model. Consider the case when the information comes from measurements of a position of one tracer and no other measurements are used. A comparison of our assimilation scheme with the nonassimilating model is illustrated in Fig. 1. Synthetic observations of tracer position [obtained by adding a random error to the true tracer position according to (33)] were taken every $\Delta T=1.0$. The full flow was simulated by the solution of the stochastic system (21) with $\sigma=0.02$. Vortices were initialized according to (42) and (43). The initial condition for the tracer in the model was $\zeta(0)=$ $0.3-0.6 i$, in the simulated truth it was picked randomly from a Gaussian distribution with $E\left[\zeta^{t}(0)\right]=\zeta(0)$, 


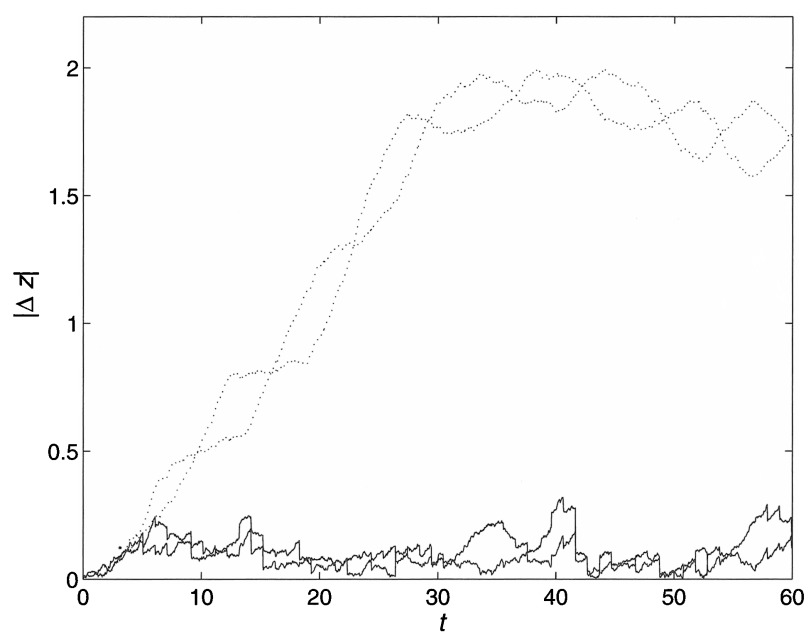

FIG. 1. EKF in a two-vortex system, one tracer is observed $\left(N_{F}=\right.$ $\left.2, N_{D}=1\right), \Delta T=1.0, \sigma=0.02, \rho=0.02$. Actual error in vortex positions $\left|z_{1,2}^{f}-z_{1,2}^{t}\right|$ in the model assimilating tracer positions (solid lines), and in the model without assimilation (dotted lines).

$E\left[|\Delta \zeta(0)|^{2}\right]=2 \rho^{2}, \rho=0.02$. Figure 1 shows the actual vortex position errors $\left|\Delta z_{1,2}(t)\right|$ in the deterministic model without assimilation and in the model that assimilates tracer position. Without assimilation the errors grow up to the system size $l$ in about 1.5T. Assimilation of the tracer position affords successful tracking of the vortices: the errors do not grow beyond $\Delta z_{\max } \approx 0.4$ for the full length of the simulation. A comparison of the predicted root-mean-square (rms) error from $\mathbf{P}^{f}$ with actual errors for a given noise realization in Fig. 2 shows a good agreement between the two.

Figure 3 shows the errors for a similar experiment with a different noise realization with $\sigma=0.05$ and $\rho$ $=0.05$. The assimilating model tracks the full system for about $3 T$ but fails after that. The expected rms errors predicted by the variance equation do not correspond to actual errors and remain misleadingly moderate. The malfunction of the filter is caused by the breakdown of the TLM. In nonlinear systems the probability density function (PDF) of the error can differ significantly from a Gaussian with the covariance predicted by (27). In this situation an update may decrease the expected error variance and at the same time deteriorate the state estimate. Our scheme would work properly only when the noise levels $\sigma$ and $\rho$ are moderate so that $\Delta \mathbf{x}$ remains small enough and the TLM remains a good approximation. This is a general feature of the EKF (see, e.g., Miller et al. 1994) rather than the result of the Lagrangian nature of observations.

To analyze the role of the system's parameters we have performed $N_{e}=100$ experiments with different noise realizations for each parameter set. The results are summarized in Table 1. The diagnostics shown in the table are $N_{f}$, the percentage of failed experiments in which $\left|\Delta z_{1,2}\right|$ exceeded the "successful tracking threshold" (chosen to be 1) for at least one step, and $d=$ (a)

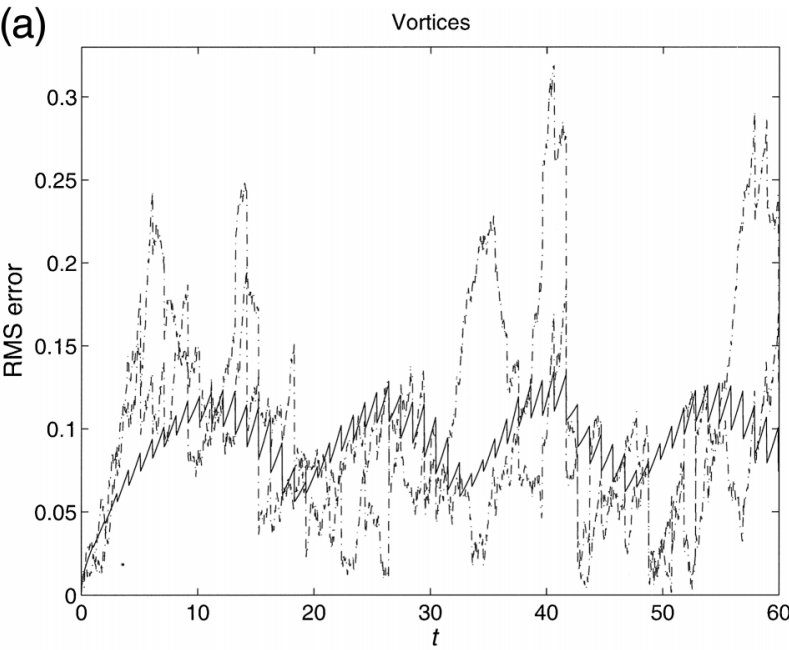

(b) Tracers

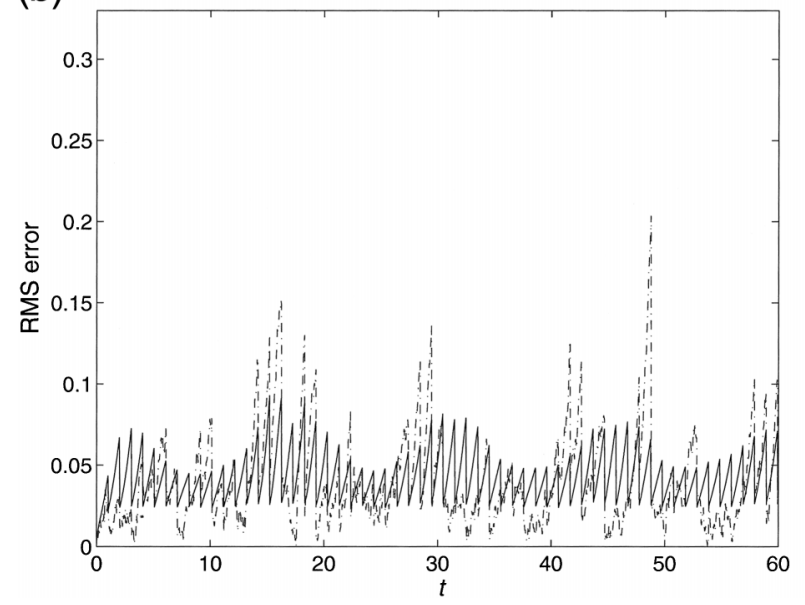

FIG. 2. Same assimilation experiment as in Fig. 1. (a) Predicted rms error in vortex location (solid) vs actual error (dashed) and (b) same for the tracer.

$\left\langle\left|\Delta z_{1,2}\right|\right\rangle$, the actual error in the predicted vortex positions averaged over time and noise realizations. The simulation time was $60(\approx 5 T)$. The scheme works for relatively large intervals between observations, the dependence on $\sigma$ is considerably stronger than the dependence on the observation error $\rho$. This is not surprising - there are no direct vortex observations and the update of their positions is carried through $\mathbf{P}_{D F}^{f}$, the predicted correlations between the vortices and the tracer [see (20)]. The accuracy of updates depends strongly on the accuracy of the prediction of $\mathbf{P}_{D F}$, which rapidly deteriorates as $\sigma$ increases.

Figure 4 illustrates results of two experiments from the table with $\rho=0.02$ and $\sigma=0.02$. For $\Delta T=1.0$ (Fig. 4a) the assimilation works well-in all 100 experiments vortex errors were well below $l$. Less frequent observations, $\Delta T=1.5$, (Fig. 4a) result in a larger error in vortex tracking.

More importantly, the filter caused a spontaneous di- 
(a)

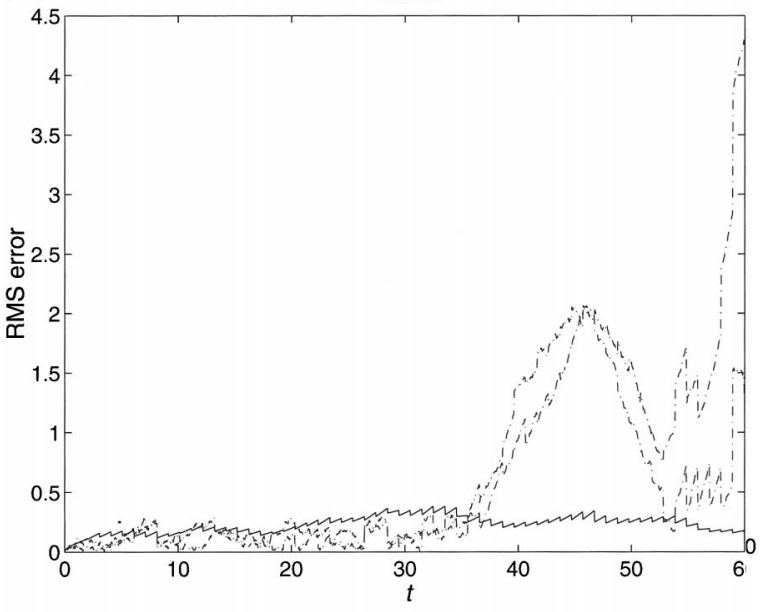

(b)

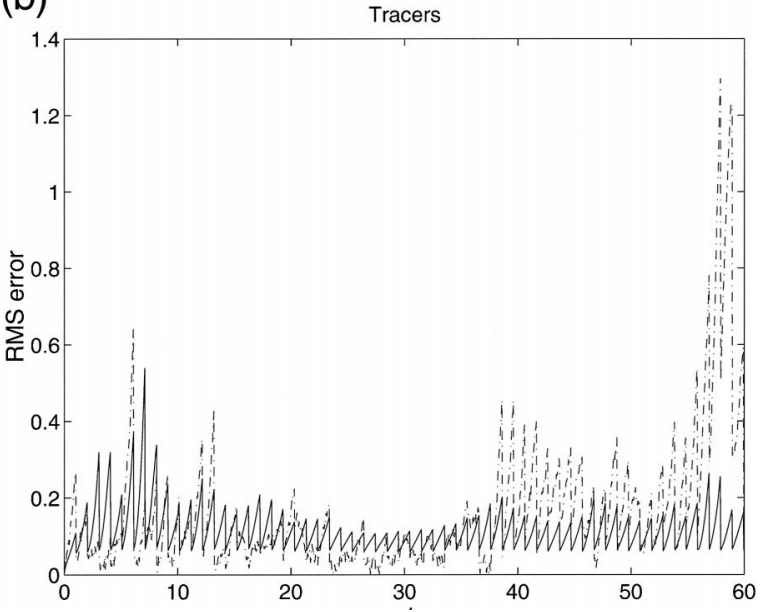

FIG. 3. Same as in Fig. 2 but $\sigma=0.05, \rho=0.05$.

vergence of the model solution in two experiments where, during one of the update steps, the $\left|\Delta z_{1,2}\right|$ jumped up to the characteristic size of the system. Such spontaneous divergence is triggered by the passage of the tracer through neighborhoods of saddle-points of the velocity field. When the tracer approaches a saddle an error in its position grows exponentially along the unstable direction and $|\Delta \zeta|$ can reach large values between two consecutive observations. The next update (a)

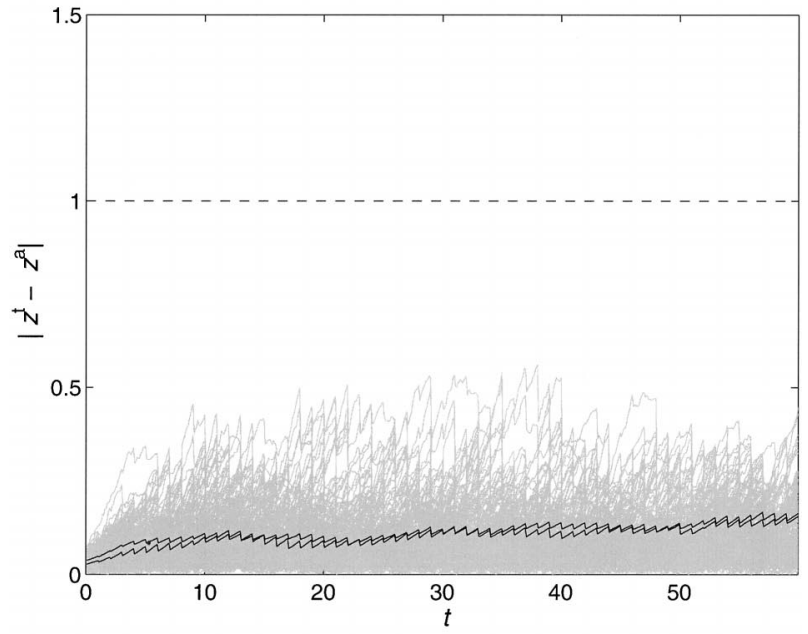

(b)

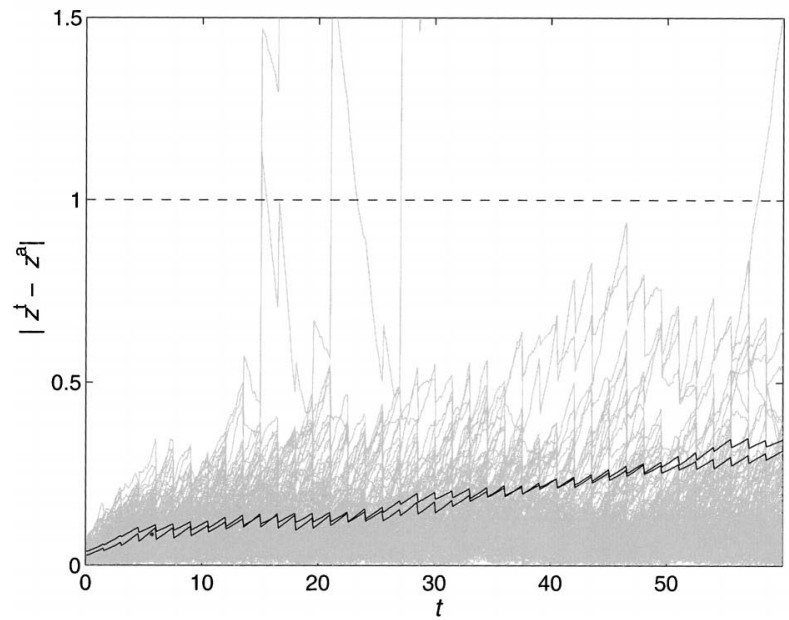

FIG. 4. Here $N_{F}=2, N_{D}=1$. Average of $\left|z_{j}^{f}-z_{j}^{t}\right|, j=1,2$ over $N_{e}=100$ experiments (black lines). Individual experiments shown in gray lines. (a) The model stays close to the full system in all 100 experiments. (b) Lower frequency of observations results in EKF failure in 2 out of 100 experiments.

will correct the tracer, which is observed directly, but it may deteriorate the estimates of the vortex positions, because these corrections are computed from the $\mathbf{P}^{f}$, and the forecast of the latter is unreliable when $|\Delta \zeta|$ is large.

TABle 1. Assimilation of the tracer position, $N_{F}=2, N_{D}=1$ (results of $N_{e}=10$ experiments with different noise realizations). Failure rate $N_{f}$ and average error in vortex positions $d$ for different noise levels $\sigma, \rho$, and for different intervals between observations $\Delta T$.

\begin{tabular}{|c|c|c|c|c|c|c|c|}
\hline & $\Delta T$ & 0.75 & 1.0 & 1.25 & 1.5 & 1.75 & 2.0 \\
\hline$\sigma=0.02$ & $N_{f}(\%)$ & 0 & 0 & 2 & 2 & 8 & 18 \\
\hline$\rho=0.02$ & $d$ & 0.09 & 0.10 & 0.12 & 0.19 & 0.35 & 0.51 \\
\hline$\sigma=0.02$ & $N_{f}(\%)$ & 0 & 1 & 7 & 19 & 22 & 29 \\
\hline$\rho=0.05$ & $d$ & 0.116 & 0.129 & 0.220 & 0.393 & 0.425 & 0.669 \\
\hline$\sigma=0.03$ & $N_{f}(\%)$ & 4 & 7 & 16 & 25 & 25 & 46 \\
\hline$\rho=0.02$ & $d$ & 0.197 & 0.289 & 0.512 & 0.775 & 0.808 & 1.13 \\
\hline
\end{tabular}




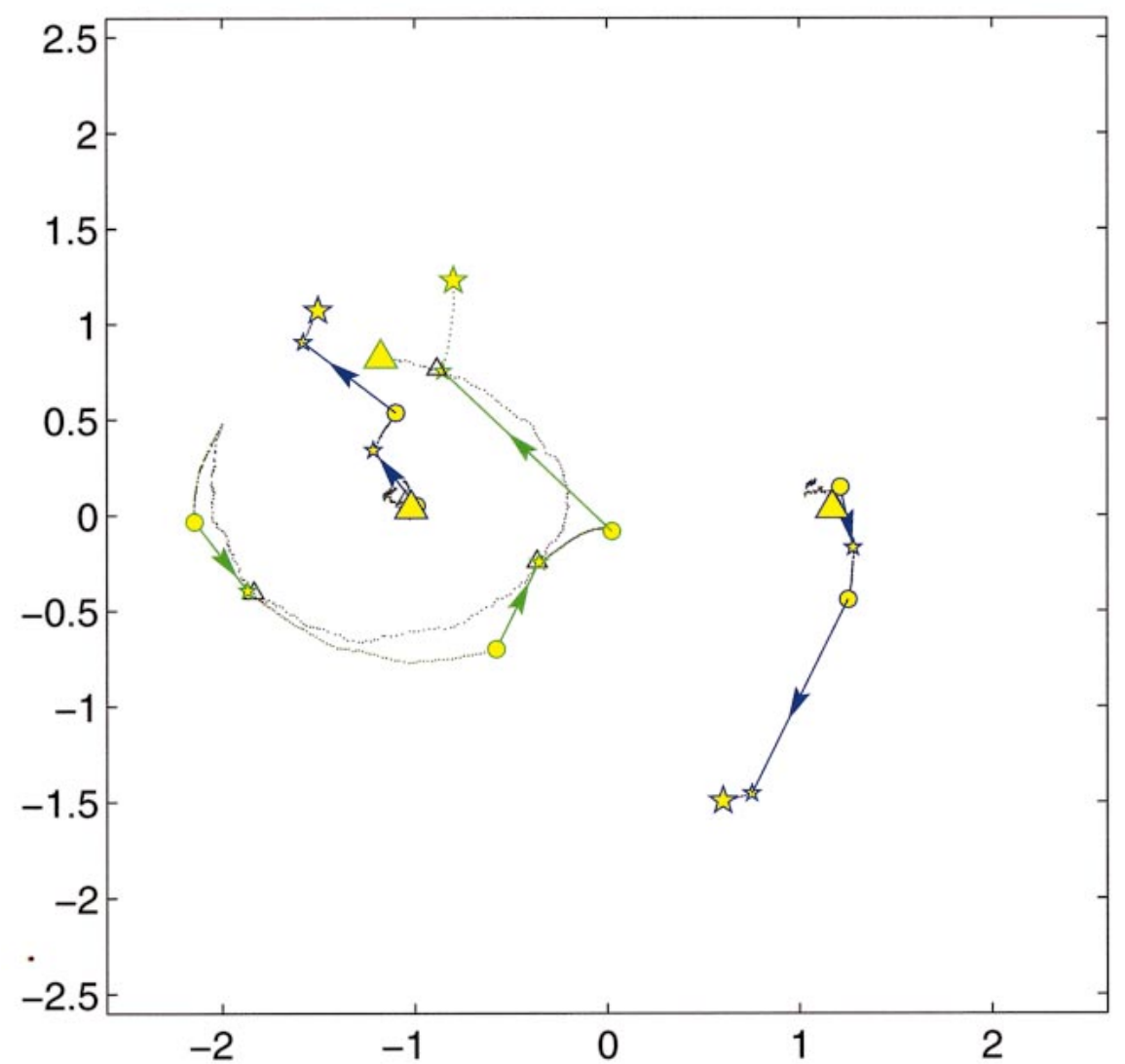

FIG. 5. Trajectories of the full system and the model in the corotating frame, shown for $8<t<$ 12.4; $\Delta T=1.5, \sigma=0.04, \rho=0.02, N_{F}=2, N_{D}=1$. Small symbols: vortex and tracer positions at the observation times $t_{j}=9,10.5,12$. Triangles: $\mathbf{x}_{j}^{t}$ (full system), circles: $\mathbf{x}_{j}^{f}$ (model before updates), stars: $\mathbf{x}_{j}^{a}$ (analysis state). Arrows: correction vectors. Large symbols: final positions at $t=12.4$. Vortices are shown in blue, tracer in green.

A typical example of the spontaneous filter divergence is shown in Fig. 5, where the trajectories of the full and the model system are plotted together in the reference frame co-rotating with the vortices. The time series of actual and expected rms errors are shown in Fig. 6. Prior to the last update shown in Fig. 5 (at $t=$ 12) the vortex error was $\left|\Delta z_{1,2}\right| \approx 0.5$, similar experiments indicate that an error of this magnitude is usually corrected by the following update with tracer data. In the present case $|\Delta \zeta|$ grew considerably faster (see Fig. $6 b)$ due to the exponential trajectory separation near the saddle. The expected error PDF (Gaussian with covariance $\mathbf{P}^{f}$ ) did not reflect the actual error distribution, and the update at $t=12$ increased the actual vortex error (Fig. 6a). The filter failed to give a correct selfestimation of its performance after the divergence: expected rms errors are almost an order of magnitude smaller than the actual ones, Fig. 6 [note the inconsistency between the innovations (crosses in Fig. 6b) and their expectations after the divergence].

Since the divergence is caused by the passage of the tracer through "bad spots" that give rise to trajectories with large separation rates, it is important to locate these spots and to find a way to treat data coming from them. A detailed discussion of these issues is beyond the scope of this paper. As a remark, we would like to mention that maps of relative dispersion that are used to study mixing in geophysical flows (Jones and Winkler 2002) seem to be an appropriate tool for the location of such regions.

\section{1) Dependence on the Launch location}

In all previous experiments the initial condition of the tracer $\zeta(0)$ was fixed. It is clear that the performance of the scheme will depend on $\zeta(0)$. If we take $|\zeta(0)|$ $\gg l$ the tracer will stay in the far region and the influence of the relative vortex motion on the tracer would be insignificant, and the updates would not provide enough constraint on the drifts of individual vortices induced by system noise. On the other hand if the tracer is taken too close to a vortex its fast rotation would lead to a rapid growth of the tracer error $\Delta \zeta$, and unless the observation frequency is substantially increased, the error 
(a)

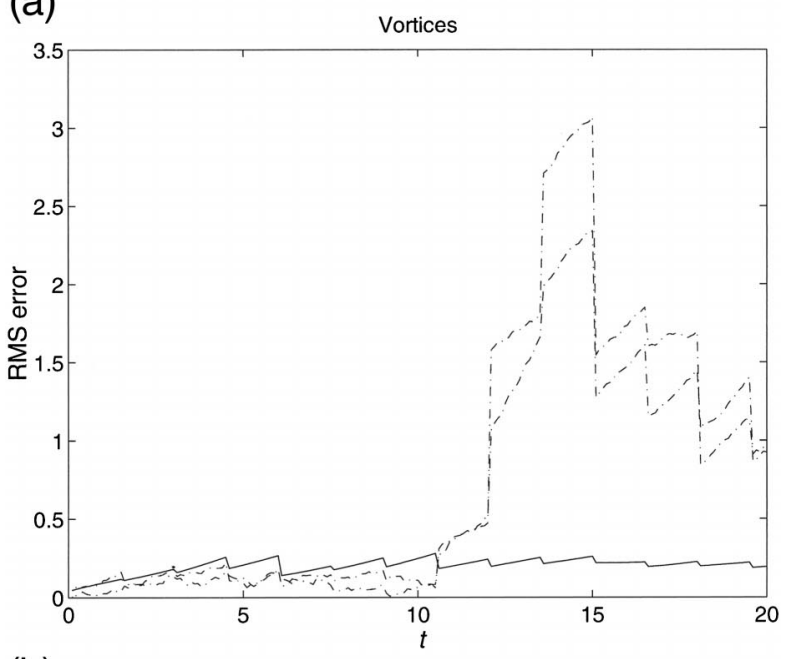

(b)

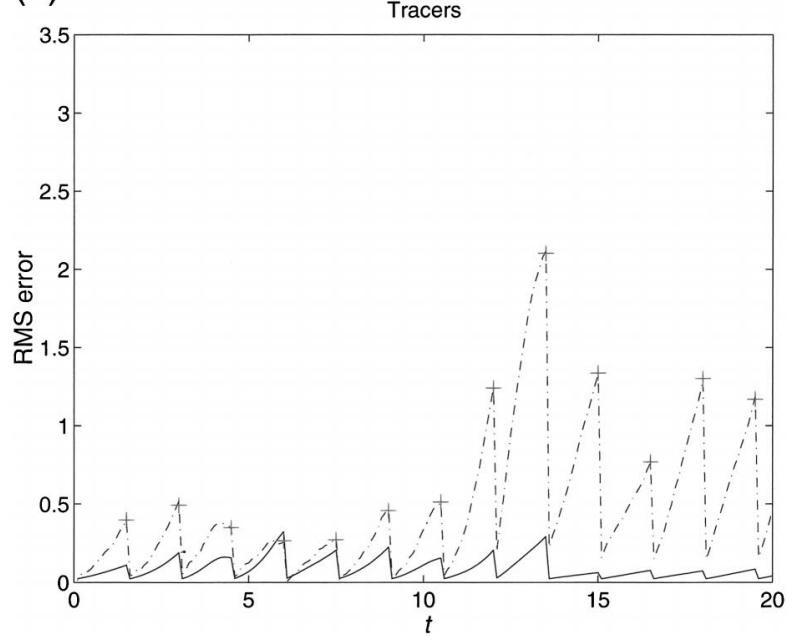

FIG. 6. (a) Actual rms errors (dashed) vs expected ones (solid) for the experiment in Fig. 5. (b) Same for the tracer, crosses correspond to the innovations $\mathrm{I}_{\mathbf{y}_{j}^{o}}-{ }_{\zeta_{j}^{f}} \mathrm{I}$.

covariance predicted by the TLM would become unreliable.

The dependence of the method's performance on $\zeta(0)$ in the case when both distances $\left|\zeta(0)-z_{1,2}(0)\right|$ are of order $l$ is non trivial (see Table 2). The error diagnostics $N_{f}$ and $d$ are defined in the same way as above, the average is over $N_{e}=200$ noise realizations. The variation in the filter performance is very strong: for our original choice of $\zeta(0)=0.3-0.6 i$ only about $1 \%$ of the 200 experiments failed $\left(N_{f}=1.5 \%\right.$ for $\rho=0.02$ and $N_{f}=0.5 \%$ for $\rho=0.04$ ), on the other hand when the tracer was launched closer to one of the vortices, $\zeta(0)=1-0.6 i$, the filter diverged in half of the experiments. Initial conditions of the tracers from Table 2 are plotted in solid circles (numbered from 1 to 5 ) in Fig. 7 on the background of the streamfunction of the two-vortex system in the corotating frame. Without noise, the tracers would follow the streamlines and their motion would be periodic. The separatrices (dark lines in Fig. 7) divide the flow plane into regions with different types of motion such as vortex cores where tracers rotate around only one vortex, a figure-eight area with tracers rotating around both vortices, two ghost vortices on each side, and the far region (outside the last separatrix). Model noise introduces time dependence into the streamline pattern, and the above description of the tracer motion becomes invalid. Tracers in the outlined regions still follow the pattern to some extent, but now they may cross the separatrices and change their motion type. It is reasonable to expect that the filter efficiency would depend on the initial condition $\zeta(0)$ relative to the corotating frame separatrices, or, in the more general case (when there is no single corotating frame in which the boundaries between regions with different motion types can be approximated by streamfunction separatrices), relative to Lagrangian coherent structures. Results in Table 2 agree with this expectation. Good launch locations yielding less than $1 \%$ filter failure rate are the ones inside the ghost vortices $(1,3$, and 5), where they neither approach the vortices too closely, nor stay far away from them. When the tracer is launched inside of the vortex core (2) the filter tends to diverge due to fast tracer rotation. In the far region (4) the updates are insignificant and do not constrain the noise-induced drift of the vortices. To see this let us define a relative distance $r=r_{z \zeta} / r_{12}$ between the tracer $\zeta$ and vortex centroid $z_{c}=z_{1}+z_{2}$, where $r_{z \zeta}=\left|\zeta-z_{c}\right|$ and $r_{12}=\mid z_{1}-$ $z_{2} \mid$ is the distance between the vortices. A far-away tracer has a large $r$ by definition. For a large $r$, the correlation terms for $z_{1}$ and $z_{2}$ with respect to $\zeta$ become indistinguishable. In this case, the filtering process can correct only $z_{c}$ but not individual positions of $z_{1}$ and $z_{2}$ that may slowly drift away from each other. This is somewhat similar to the case without assimilation shown in Fig. 1, but $z_{c}$ is estimated correctly by the faraway $\zeta$.

TABLE 2. Filter failure rate $N_{f}$ and average error in vortex position $d$ for different tracer initial conditions $\zeta(0)$ (see Fig. $\left.7 \mathrm{a}\right) ; N_{F}=2$, $N_{D}=1, \Delta T,=1.0 ; N_{f}$ and $d$ same as in Table 1.

\begin{tabular}{lccccc}
\hline \hline & $\zeta(0)$ & $0.3-0.6 i$ & $1-0.6 i$ & $1-i$ & $2.4-2.4 i$ \\
\hline$\sigma=0.02$, & $N_{f}(\%)$ & 1.5 & 41 & 0 & 4 \\
$\rho=0.02$ & $d$ & 0.14 & 1.63 & 0.09 & 0 \\
$\sigma=0.02$, & $N_{f}(\%)$ & 0.5 & 55.5 & 0.5 & 0.20 \\
$\rho=0.04$ & $d$ & 0.12 & 1.90 & 0.11 & 0 \\
\hline
\end{tabular}


(a)

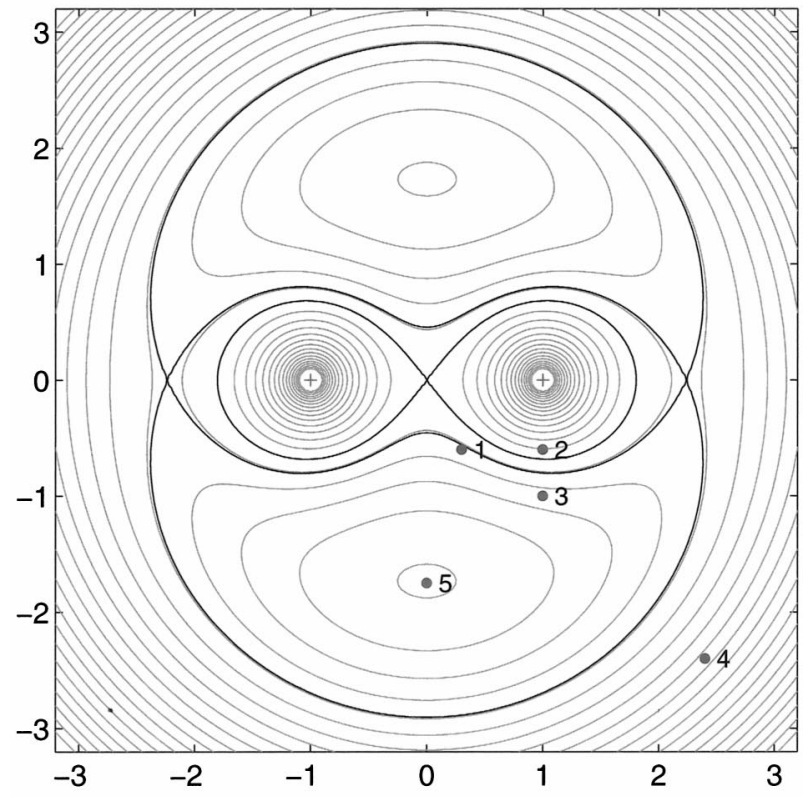

(b)

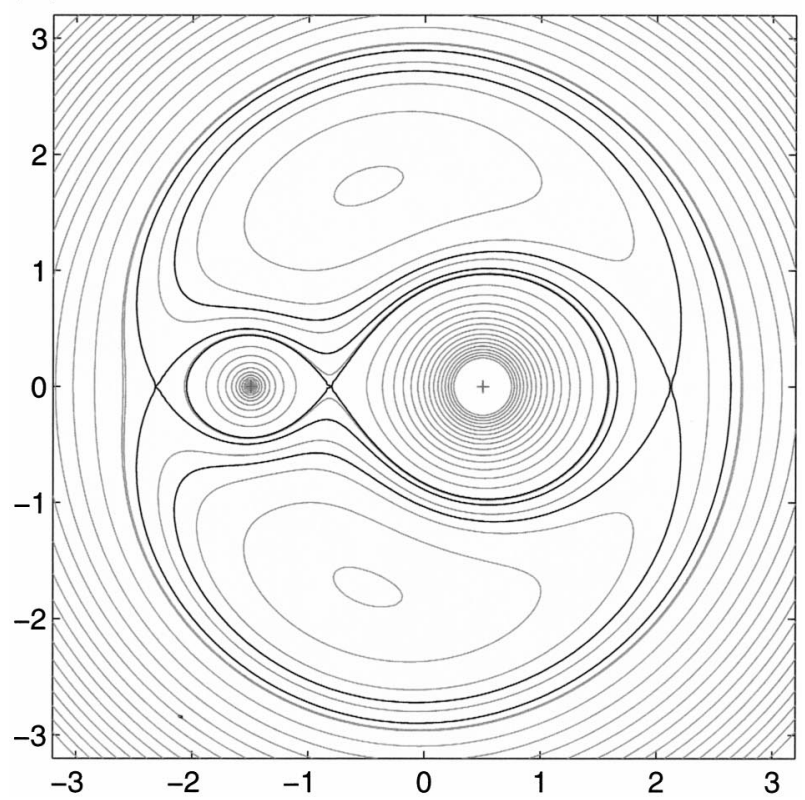

FIG. 7. Streamfunction of the flow due to two-point vortices in the corotating frame: (a) $\Gamma_{1}=\Gamma_{2}$; (b) $3 \Gamma_{1}=\Gamma_{2}$.

A more detailed analysis of dependence of the efficiency of the assimilation on $\zeta(0)$ is presented in Fig. 8. Two different vortex systems were considered: with $\Gamma_{1}=\Gamma_{2}=2 \pi$ (same as in all previous experiments) (Fig. 8a) and with $3 \Gamma_{1}=\Gamma_{2}=1.5$ (Fig. 8b). Streamline patterns in the corotating frame are shown in Fig. 7. The parameters are $\sigma=0.02, \rho=0.04$, and $\Delta T=1.0$, tracers were launched on the $y$ axis: $\zeta(0)=i y_{0}$. The (a)

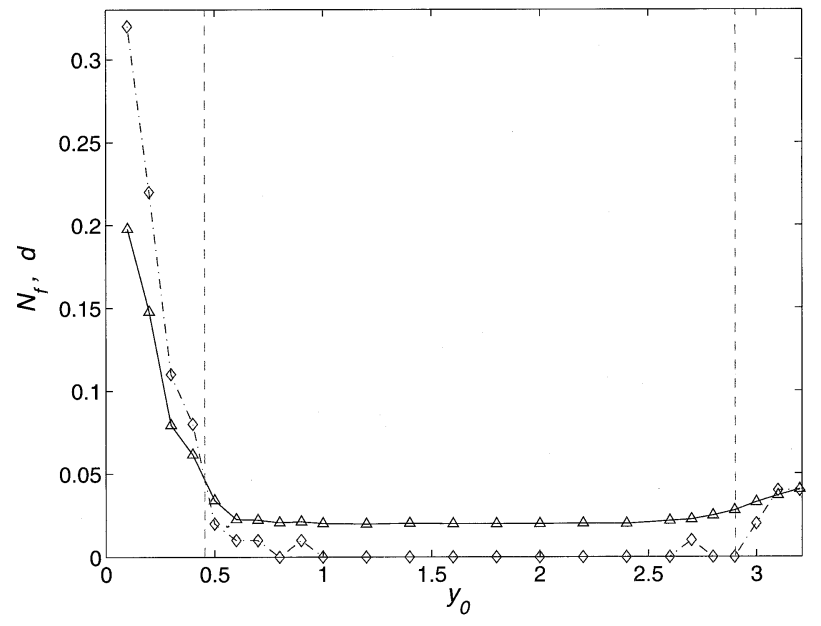

(b)

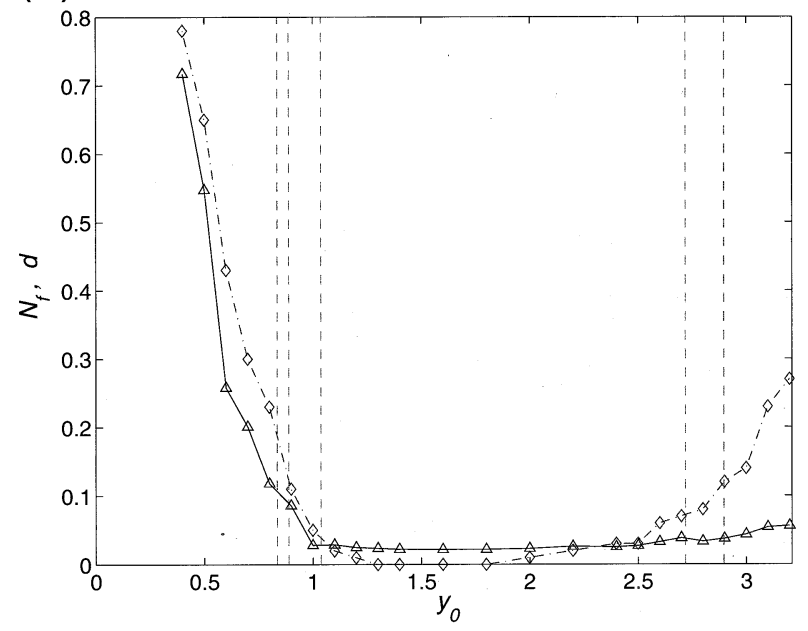

FIG. 8. Dependence of the filter failure rate $N_{f}$ (diamonds) and average error in vortex position $d$ (triangles) on tracer launch location, $\zeta(0)=i y_{0}$. Abscissa: $y_{0}$-position of the tracer on the vertical; ordinate: $N_{f}$ (dimensionless) and $d$ (in units of distance). (a) $\Gamma_{1}=\Gamma_{2}, y_{0} \in$ $[0.1,3.2]$; (b) $3 \Gamma_{1}=\Gamma_{2}, y_{0} \in[0.4,3.2]$. Vertical lines mark separatrix locations (see Fig. 7).

plots show the $N_{f}$ (diamonds) and $d$ (triangles) as functions of $y_{0}$. The error diagnostics exhibit strong variation around the separatrix values of $y_{0}$-the effect of the flow structures on the filter performance is evident in both cases.

Understanding the correlation between the informational value of a drifter and its initial position is a crucial step in devising optimal launching strategies. Lagrangian flow structures provide a template that divides the flow into regions with different types of tracer motion. Our results show that the performance of the assimilation scheme depends on which structure the drifter was launched into, and that drifters in the same structure yield similar results (e.g., the plateaus in Figs. 8a,b corresponding to the ghost vortices). 
TABLE 3. Assimilation of the flow velocity approximated at the tracer location by a second-order finite difference (45); $N_{F}=2, N_{D}$ $=1, \sigma=0.02, \rho=0.02$.

\begin{tabular}{cllcc}
\hline \hline$\Delta T$ & 0.75 & 1.0 & 1.25 & 1.5 \\
\hline$N_{f}(\%)$ & 0 & 7 & 27 & 64 \\
$d$ & 0.18 & 0.30 & 0.39 & 0.64 \\
\hline
\end{tabular}

\section{2) COMPARison to velocity ASSimilation}

An alternative approach to the assimilation of Lagrangian information is to estimate the velocity of the flow at the drifter location from two (or more) consecutive position observations. The flow velocity is an Eulerian variable that has a straightforward expression in terms of the flow state vector. In this case there is no need to track tracers and corresponding covariances, which results in smaller system dimension; on the down side, the quality of the observations deteriorates (additional errors are introduced by finite differences).

To compare velocity assimilation with our approach we have performed simulations in the same setting as before: the system was initialized according to (42) and (43) and was evolved by (21) and (22), where the covariance of the system noise $\boldsymbol{\eta}$ was given by (31). Tracer positions were observed every $\Delta T$ [with error covariance given by (40)] and a second-order finite difference was used to estimate the flow velocity $\mathbf{v}\left(\mathbf{y}_{j}^{o}\right)$ at observed tracer locations $\mathbf{y}_{j}^{o \text { : }}$

$$
\mathbf{v}_{j}\left(\mathbf{y}_{j}^{O}\right)=\left(3 \mathbf{y}_{j}^{O}-4 \mathbf{y}_{j-1}^{O}+\mathbf{y}_{j-2}^{O}\right) /(2 \Delta T)+\tilde{\boldsymbol{\epsilon}}_{j},
$$

where $\tilde{\boldsymbol{\epsilon}}_{j}$ denotes the error resulting from the tracer observation error $\boldsymbol{\epsilon}$, model noise $\boldsymbol{\eta}$, and finite $\Delta T$. We approximate $\tilde{\boldsymbol{\epsilon}}_{j}$ as [neglecting $O\left(\eta \Delta T, \Delta T^{3}\right)$ terms]

$$
\begin{aligned}
\tilde{\boldsymbol{\epsilon}}_{j}= & \left(3 \boldsymbol{\epsilon}_{j}-4 \boldsymbol{\epsilon}_{j-1}+\boldsymbol{\epsilon}_{j-2}\right) /(2 \Delta T)+\boldsymbol{\eta}_{j-1} \sqrt{\Delta T} \\
& +\boldsymbol{\eta}_{j-2} \sqrt{2 \Delta T},
\end{aligned}
$$

that results in

$$
\tilde{\mathbf{R}}=\left[\begin{array}{cc}
\left(\tilde{\boldsymbol{\epsilon}}_{j} \tilde{\boldsymbol{\epsilon}}_{j}^{\mathrm{T}}\right) & \left(\tilde{\boldsymbol{\epsilon}}_{j} \tilde{\boldsymbol{\epsilon}}_{j}^{* \mathrm{~T}}\right) \\
\left(\tilde{\boldsymbol{\epsilon}}_{j}^{* \mathrm{~T}} \tilde{\boldsymbol{\epsilon}}_{j}\right) & \left(\tilde{\boldsymbol{\epsilon}}_{j}^{*} \tilde{\boldsymbol{\epsilon}}_{j}^{* \mathrm{~T}}\right)
\end{array}\right]=\left(\frac{13}{2}\right) \frac{\mathbf{R}}{\Delta T^{2}}+\left(\frac{5}{2}\right) \frac{\mathbf{Q}}{\Delta T} .
$$

Note that as $\Delta T \rightarrow 0$ the signal-to-noise ratio in velocity observations goes to zero, because the tracer displacement due to the noise scales as $\sqrt{\Delta T}$, while the contribution of the deterministic part is $\sim \Delta T$ (as a result $\tilde{\mathbf{R}}$ diverges as $\Delta T \rightarrow 0)$.

The velocities (45) are assimilated into the vortex model via the EKF. The observation function $h_{j}(\mathbf{z})$ is given by the expression for the velocity induced by the vortices at observed tracer locations:

$$
h_{j}(\mathbf{z})=\sum_{m=1}^{N_{F}} \frac{i \Gamma_{m}}{\mathbf{y}_{j}^{O *}-z_{m}^{*}},
$$

it is nonlinear and depends on time through $\mathbf{y}_{j}^{o}$. The correlations between consecutive velocity observation errors were neglected.

The results are presented in Table 3. Comparison with
TABLE 4. Velocity assimilation. Failure rate $N_{f}$ and average error in vortex position $d$ for different tracer initial conditions $\zeta(0)$, same parameters as in Table $2(\Delta T=1, \sigma=0.02, \rho=0.02)$.

\begin{tabular}{cccccc}
\hline \hline$\zeta(0)$ & $0.3-0.6 i$ & $1-0.6 i$ & $1-i$ & $2.4-2.4 i$ & $-1.75 i$ \\
\hline$N_{f}(\%)$ & 7 & 99 & 4 & 3 & 0 \\
$d$ & 0.29 & 4.15 & 0.25 & 0.21 & 0.16 \\
\hline
\end{tabular}

Table 1 shows that assimilation with the augmented model is more accurate and has lower failure rates $\left(N_{f}\right)$. The dependence of the efficiency of the velocity assimilation on the tracer initial position (Table 4) shows the same character as in the case of position assimilation. The difference in the performance of the two schemes is pronounced when the tracer is launched close to the vortices; for the tracer in the far region, $\zeta(0)=2.4-$ $2.4 i$, both schemes give similar results.

\section{b. Four vortices}

Depending on vortex circulations and initial positions the motion of a deterministic four-vortex system can be (quasi) periodic, chaotic, or can reach a singularity in finite time (Aref and Pomphrey 1982; Novikov and Sedov 1978; Ziglin 1980; Kimura 1990). We will consider a system of four identical vortices, $\Gamma_{m}=2 \pi, m=1$, $\ldots, 4$, and assess the performance of our scheme in case when the deterministic model can possess chaotic vortex motion (the existence of regions of strong local instability, which is a precursor of chaotic dynamics, is known to have a negative effect on the EKF performance (Miller et al. 1994). For our choice of $\sigma, \rho$, and $\Delta T$, we found that one tracer cannot provide enough information to estimate four vortices: failure rates are close to 1 in numerical experiments with $\left(N_{F}, N_{D}\right)=$ $(4,1)$. Here we report experiments with $\left(N_{F}, N_{D}\right)=(4$, 2).

We start from a vortex motion that does not visit the unstable part of the phase space by initializing vortices at the corners of a square: $z_{m}(0)= \pm \sqrt{3 / 2}(1 \pm i)$. The length scale is chosen in such a way that the period of the rotation of the unperturbed system, $T=4 \pi$, coincides with that of the vortex pair in section $4 \mathrm{a}$. Two tracers are initialized at $\zeta_{1}=2.5 i$ and $\zeta_{2}=0.5$. Vortex and tracer initial conditions have uncorrelated errors with $E\left[\Delta z_{m}(0) \Delta z_{m}^{*}(0)\right]=E\left[\Delta \zeta_{l}(0) \Delta \zeta_{l}^{*}(0)\right]=2 \rho^{2}, m=$ $1, \ldots, 4, l=1,2$. Figure 9 shows an experiment with $\sigma=0.02$ and $\rho=0.02$, the tracers were observed every $\Delta T=1.0$. Similarly to the two-vortex system, the model without assimilation loses track of the full system after a couple of rotations. The errors of the assimilating model remain small during the entire simulation, a comparison with their predicted values, $\left(\operatorname{tr} \mathbf{P}_{2 F F}^{f} / N_{F}\right)^{1 / 2}$, in Fig. $10 \mathrm{a}$ shows the agreement between the two. We have repeated the experiment with different noise realizations and found that our assimilation scheme works very well. In this example, the four-vortex system was initialized in the "middle" of its stability region. Figure 10b shows 


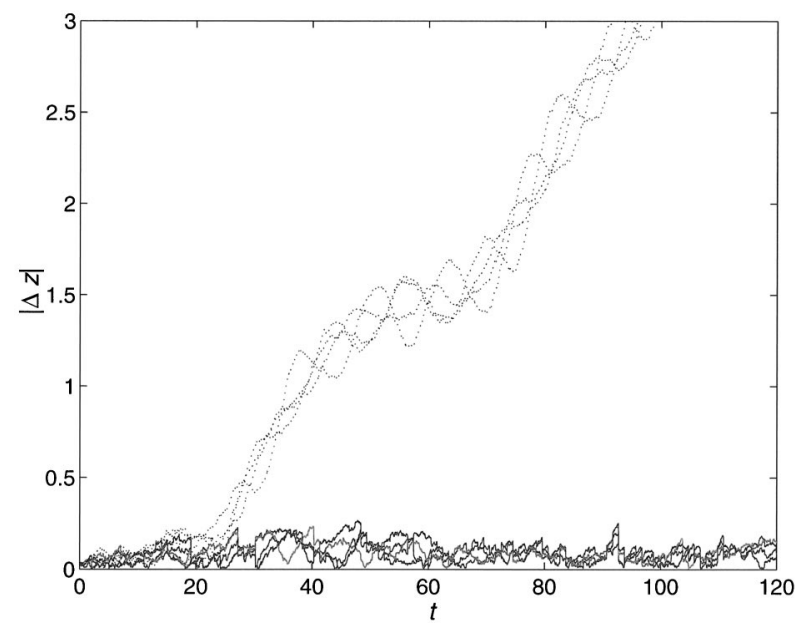

FIG. 9. The $\left|z_{m}^{f}-z_{m}^{t}\right|, m=1, \ldots, 4$, symmetric vortex initial conditions, $z_{j}(0)= \pm \sqrt{(3 / 2)}(1 \pm i) ; \Delta T=1.0, \sigma=0.02, \rho=0.02$, $N_{F}=4, N_{D}=2$. Dotted: no assimilation. Solid: assimilation of tracer position.

the time series of the absolute value of vortex (solid) and tracer (dashed) velocities [i.e., the deterministic part of the right-hand sides of equations (21) and (22)], it is evident that the vortices never deviated far from a table synchronous rotation.

In our next example, vortices were initialized at $z_{m}(0)$ $= \pm 4 \pm i$ (corners of a rectangle), corresponding to a quasi-periodic motion. Tracers were launched at $\zeta_{1}(0)$ $=-0.5+i$ and $\zeta_{2}(0)=0.5$, observation frequency and noise parameters were the same as above. The actual vortex position errors (dashed) and the predicted rms error (solid) are plotted in Fig. 11a. The latter is just slightly larger than in the previous experiment, but the actual errors are sometimes tripled. Nevertheless, the model was able to track the full system through the entire observation period. Vortex and tracer velocities are plotted in Fig. 11b. Vortex motion can be described as a sequence of noise-induced transitions between different quasi-periodic regimes. The unstable dynamics of the underlying deterministic system results in larger tracking error, despite the fact that the motion is slower (absolute values of vortex velocities in Fig. 11b are smaller than in Fig. 10).

Figure 12 shows results of a similar experiment with the vortices initialized in the chaotic part of the phase space of the deterministic model, $\mathbf{z}(0)=(5.241+$ $3.609 i,-2.1213,2.1213,-3 i)$. The rms error in Fig. 12a shows that the system was tracked through the entire observation period, but the errors were consistently larger than in the previous experiments. Vortex velocities (Fig. 12b) are of the same scale as in the previous examples, but their time series have irregular appearance typical of chaotic motion. Similar experiments with different noise realizations show that the performance of the filter in chaotic regime is irregular and to ensure successful tracking the noise levels must be reduced.

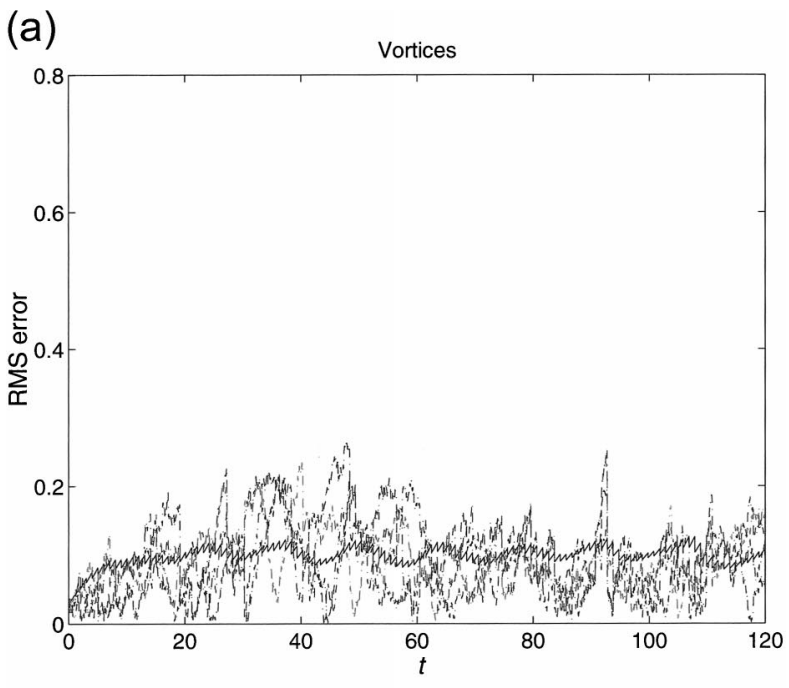

(b)

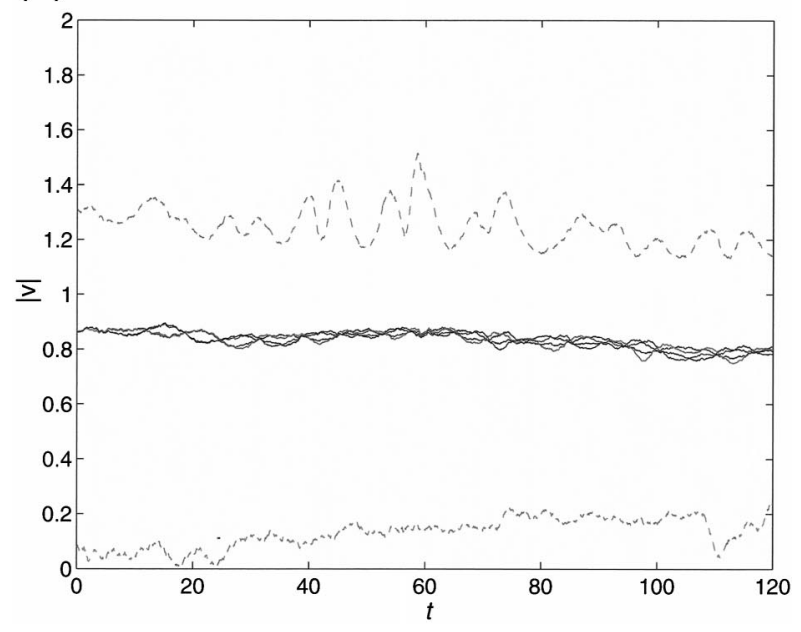

FIG. 10. Same experiment as in Fig. 9. (a) Solid: expected vortex rms; dashed: actual error. (b) Solid: vortex velocities $\left|\dot{z}_{m}\right|, m=1$, $\ldots, 4$; dashed: tracer velocities $\left|\dot{\zeta}_{l}\right|, l=1,2$.

For example, in the above experiment the filter worked reasonably well for $0<t<60$ (with $N_{f}=4 \%$ ), but diverged in $N_{f}=40 \%$ of experiments on $0<t<120$.

We have kept the observation frequency and noise parameters fixed in all these experiments, varying the system length scale $l$ in order to control the performance of the assimilating model. Smaller $l$ results in a faster system, which is equivalent to larger $\Delta T$ and $\rho$, according to the scaling (41). In order to achieve successful tracking, the quasi-periodic and chaotic initializations required taking relatively large $l$ that leads to a considerably slower vortex motion. Although the parameter space of the problem is too large for generalizations (e.g., there is a strong dependence on the tracer initial conditions), it appears that chaotic vortex trajectories that spend the longest time in the unstable parts of the phase space are the most difficult to track. 
(a)

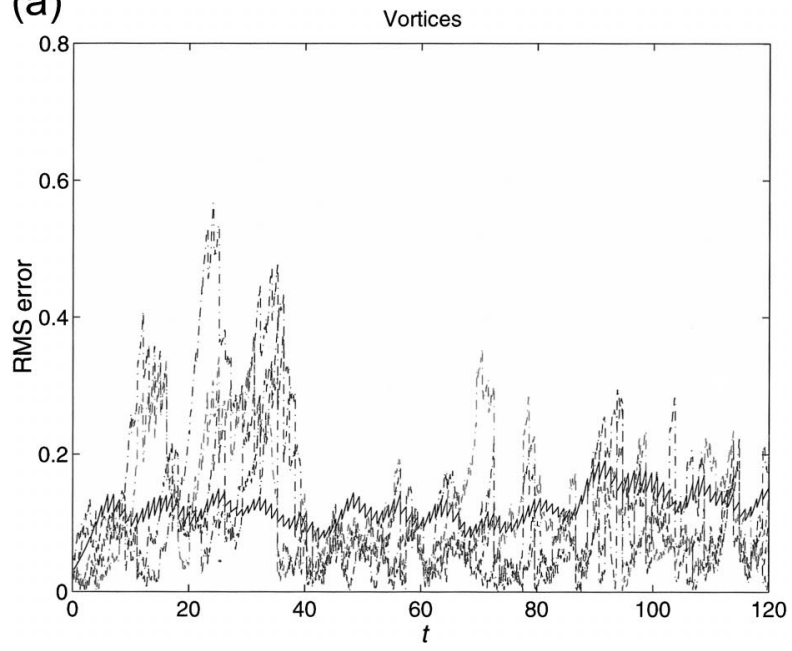

(b)

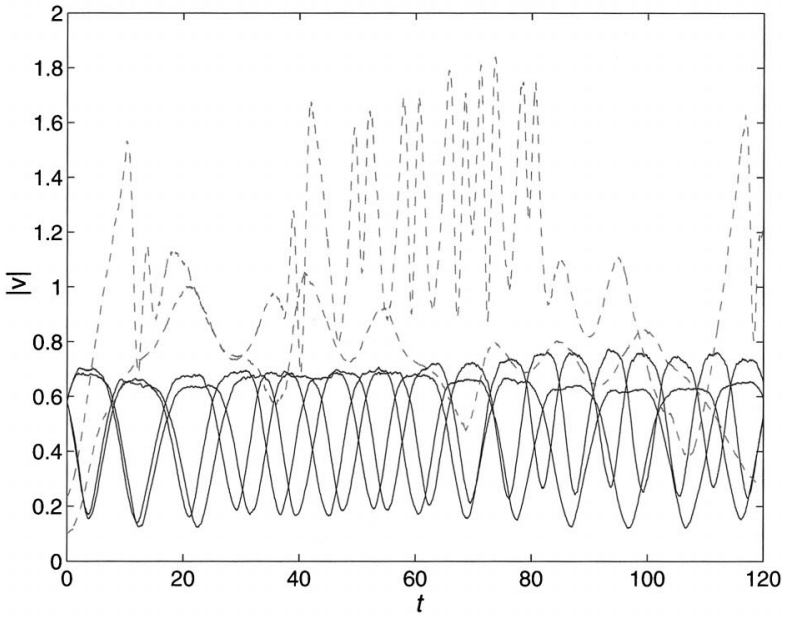

FIG. 11. Same as in Fig. 10 but vortices were initialized at the vertexes of a rectangle $z_{m}(0)= \pm 4 \pm i$; tracers at $\zeta_{1}(0)=-0.5+$ $i, \zeta_{2}(0)=0.5$.

\section{Conclusions}

We have presented a new method for the assimilation of tracer data into dynamical flow models. The main difficulty in assimilating Lagrangian data is due to the fact that drifter observations yield time-integrated information that is not a function of an instantaneous flow state. We overcome this problem by augmenting the model, treating tracer coordinates as additional state vector components. Information from Lagrangian observations propagates into the flow state through the error correlation between the tracer positions and the flow state.

The assimilation of tracer data into two- and fourpoint vortex systems affords successful tracking of the vortices, provided that the observations are sufficiently frequent and accurate. The filter diverges when the noise (a)

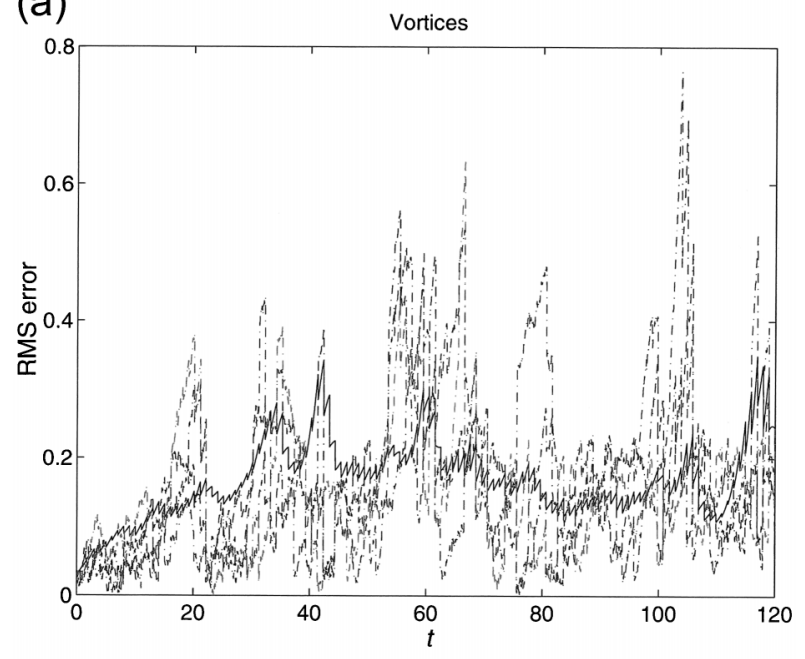

(b)

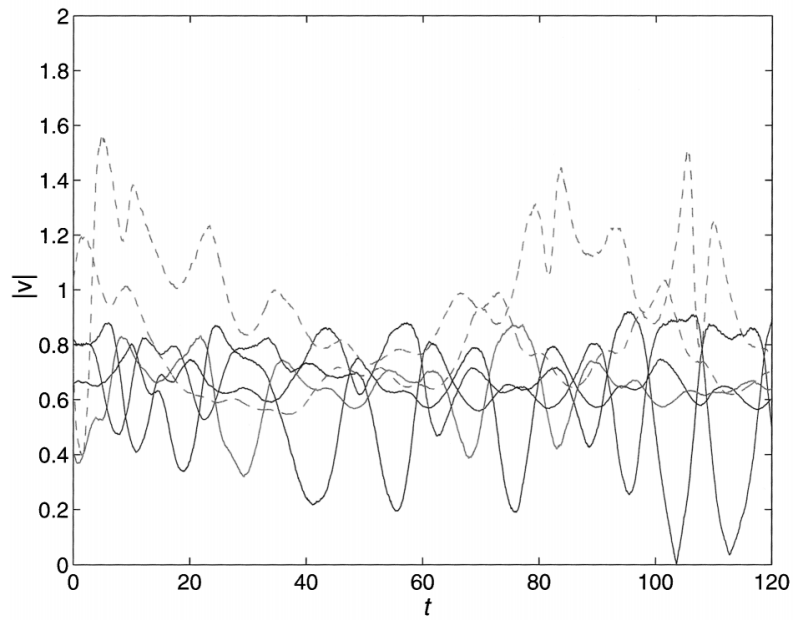

FIG. 12. Same as in Fig. 11 but for chaotic vortex initial conditions, $\mathbf{z}(0)=(5.2+3.6 i,-2.1,2.1,-3 i)$.

levels are increased accounting for the nonlinear effects that are neglected by the EKF.

The performance of the assimilation scheme strongly depends on the initial position of the tracer relative to the Lagrangian structures of the flow. In the case of two vortices the boundaries of these structures can be roughly approximated by the separatrices of the streamfunction in the corotating frame. Numerical examples confirm the strong correlation between the efficiency of the assimilation and the tracer position with respect to the separatrices.

Comparison of the proposed method with an alternative approach that assimilates flow velocity estimated from consecutive tracer positions shows that our method works better because we take into account the Lagrangian nature of drifter information.

Numerical simulations with four-point vortices demonstrate the feasibility of tracking multivortex systems 
with few tracer observations assimilated by our method. The performance of the assimilation depends not only on the scales of motion and noise levels, but on the character of the underlying deterministic vortex dynamics, or, from a different viewpoint, on the trajectory separation rates in the parts of the phase space visited during the motion. Chaotic vortex initial conditions resulted in the biggest tracking errors, whereas the tracking in case of the symmetric initialization (that restricted the motion to a stable part of the phase space) was the most accurate.

Acknowledgments. L. Kuznetsov and C.K.R.T. Jones were supported by Office of Naval Research Grant N00014-93-1-0691; K. Ide was supported by Office of Naval Research Grant N00014-99-0020.

\section{REFERENCES}

Anderson, J. L., 2001: An ensemble adjustment Kalman filter for data assimilation. Mon. Wea. Rev., 129, 2884-2903.

Aref, H., and N. Pomphrey, 1982: Integrable and chaotic motion of four vortices. I. The case of identical vortices. Proc. Roy. Soc. London, 380, 359-387.

Babiano, A., G. Boffetta, A. Provenzale, and A. Vulpiani, 1994: Chaotic advection in point vortex models and two-dimensional turbulence. Phys. Fluids, 6, 2465-2474.

Bower, A. S., and H. D. Hunt, 2000: Lagrangian observations of the deep western boundary current in the North Atlantic Ocean. Part I: Large-scale pathways and spreading rates. J. Phys. Oceanogr., 30, 764-783.

Carr, M. E., and H. T. Rossby, 2001: Pathways of the North Atlantic current from surface drifters and subsurface floats. J. Geophys. Res., 106C, 4405-4419.

Carter, E. F., 1989: Assimilation of Lagrangian data into numerical models. Dyn. Atmos. Oceans, 13, 335-348.

Cohn, S. E., 1993: Dynamics of short-term univariate forecast error covariances. Mon. Wea. Rev., 121, 3123-3149.

Dutkiewicz, S., L. Rothstein, and T. Rossby, 2001: Pathways of crossfrontal exchange in the North Atlantic current. J. Geophys. Res., 106C, 26 917-26928.

Evensen, G., 1994: Sequential data assimilation with a nonlinear quasi-geostrophic model using Monte-Carlo methods to forecast error statistics. J. Geophys. Res., 99C, 10 143-10 162.

- - 1997: Advanced data assimilation for strongly nonlinear dynamics. Mon. Wea. Rev., 125, 1342-1354.

Friedrichs, K. O., 1966: Special Topics in Fluid Dynamics. Gordon and Breach, $177 \mathrm{pp}$.

Garfield, N., M. E. Maltrud, C. A. Collins, T. A. Rago, and R. G. Paquette, 2001: Lagrangian flow in the California undercurrent, an observation and model comparison. J. Mar. Syst., 106, 201220.

Ghil, M., and P. Malanotte-Rizzoli, 1991: Data assimilation in meteorology and oceanography. Advances in Geophysics, Vol. 33, Academic Press, 141-226.

Heemink, A., M. Verlaan, and A. Segers, 2001: Variance reduced ensemble Kalman filtering. Mon. Wea. Rev., 129, 1718-1728.

Ide, K., and M. Ghil, 1998: Extended Kalman filtering for vortex systems. Part I: Methodology and point vortices. Dyn. Atmos. Oceans, 27, 301-332.

- - , P. Courtier, M. Ghil, and A. Lorenc, 1997: Unified notation for data assimilation: Operational, sequential and variational. J.Meteor. Soc. Japan, 75, 181-189.

- - , L. Kuznetsov, and C. K. R. T. Jones, cited 2002: Lagrangian data assimilation for point vortex systems. J. Turbul. [Available online at http://www.iop.org/EJ/article/-search $=3716994.1 /$ 1468-5248/3/1/053/jt2153.pdf.]

Jazwinski, A. H., 1970: Stochastic Processes and Filtering Theory. Academic Press, 376 pp.

Jones, C. K. R. T., and S. Winkler, 2002: Invariant manifolds and Lagrangian dynamics in the ocean and atmosphere. Handbook of Dynamical Systems, B. Fiedler, Ed., Vol. 2, North-Holland, $55-92$.

Kimura, Y., 1990: Parametric motion of complex-time singularity toward real collapse. Physica D, 46, 439-448.

McWilliams, J. C., 1990: The vortices of two-dimensional turbulence. J. Fluid Mech., 219, 361-385.

Miller, R. N., M. Ghil, and F. Gauthiez, 1994: Advanced data assimilation in strongly nonlinear dynamical systems. J. Atmos. Sci., 51, 1037-1056.

- - , E. F. Carter, and S. T. Blue, 1999: Data assimilation into nonlinear stochastic models. Tellus, 51A, 167-194.

Molcard, A., L. I. Piterbarg, A. Griffa, T. M. Özgökmen, and A. J. Mariano, 2003: Assimilation of drifter positions for the reconstruction of the Eulerian circulation field. J. Geophys. Res., in press.

Novikov, E. A., and Y. B. Sedov, 1978: Stochastic properties of a four-vortex system. Sov. Phys. JETP, 48, 440-444.

Özgökmen, T. M., A. Griffa, A. J. Mariano, and L. I. Piterbarg, 2000: On the predictability of Lagrangian trajectories in the ocean. $J$. Atmos. Oceanic Technol., 17, 366-383.

Pham, D. T., 2001: Stochastic methods for sequential data assimilation in strongly nonlinear systems. Mon. Wea.Rev., 129, 1194-1207.

Poje, A. C., M. Toner, A. D. Kirwan, and C. K. R. T. Jones, 2002 Drifter launch strategies based on Lagrangian templates. J. Phys. Oceanogr., 32, 1855-1869.

Verlaan, M., and A. W. Heemink, 2001: Nonlinearity in data assimilation applications: A practical method for analysis. Mon. Wea Rev., 129, 1578-1589.

Weiss, J. B., A. Provenzale, and J. C. McWilliams, 1998: Lagrangian dynamics in high-dimensional point-vortex systems. Phys. Fluids, 10, 1929-1941.

Ziglin, S. L., 1980: Nonintegrability of a problem on the motion of four point vortices. Sov. Math. Dokl., 21, 296-299. 